\title{
Divided attention: An undesirable difficulty in memory retention
}

\author{
Nicholas Gaspelin • Eric Ruthruff • Harold Pashler
}

Published online: 21 May 2013

(C) Psychonomic Society, Inc. 2013

\begin{abstract}
How can we improve memory retention? A large body of research has suggested that difficulty encountered during learning, such as when practice sessions are distributed rather than massed, can enhance later memory performance (see R. A. Bjork \& E. L. Bjork, 1992). Here, we investigated whether divided attention during retrieval practice can also constitute a desirable difficulty. Following two initial study phases and one test phase with Swahili-English word pairs (e.g., vuvi-snake), we manipulated whether items were tested again under full or divided attention. Two days later, participants were brought back for a final cued-recall test (e.g., vuvi-?). Across three experiments (combined $N=122$ ), we found no evidence that dividing attention while practicing retrieval enhances memory retention. This finding raises the question of why many types of difficulty during practice do improve long-term retention, but dividing attention does not.
\end{abstract}

\section{Keywords Divided attention $\cdot$ Memory $\cdot$ Desirable} difficulties

Unused memories are often forgotten. For example, one might find it difficult to recall a childhood street address or a password on an old Internet account. In both of these examples, the information was once frequently used and easy to recall but, following months or years of disuse, has become difficult to retrieve. Forgetting is also a major problem in educational contexts, where students are unable to retrieve learned material even just a few hours or days later (see, e.g., Schmidmaier, Ebersbach, Schiller, Hege, Holzer,

N. Gaspelin $(\varangle) \cdot$ E. Ruthruff

Department of Psychology, University of New Mexico,

MSC03 2220, Albuquerque, NM 87131-1161, USA

e-mail: gaspelin@unm.edu

H. Pashler

Department of Psychology, University of California-San Diego,

La Jolla, CA, USA
\& Fischer, 2011, on forgetting by medical students). Although rehearsal of material can protect somewhat against forgetting, it is often insufficient. Understanding how to efficiently improve long-term retention would greatly benefit society, helping us to design more effective training and education programs. R. A. Bjork and Bjork (1992) offered one influential proposal with profound implications: The key to successful long-term retention is encountering difficulty during memory retrieval. This counterintuitive hypothesis, called the desirable difficulties hypothesis, is an empirical generalization supported by a diverse set of studies using many different manipulations of difficulty in many different contexts.

Several different desirable difficulties have been reported, including spacing rather than massing practice, testing rather than restudying, and randomly mixing material types rather than blocking. In the present study, we investigated an alternative means of increasing task difficulty without the inconvenience of requiring that participants wait before restudying material. It is well-documented that people have difficulty performing two tasks concurrently. The desirable difficulties hypothesis suggests that dividing attention during retrieval practice could actually improve memory retention, even while impairing performance during practice itself. Before describing our experimental approach, we review the desirable difficulties hypothesis and previous attempts to test it.

\section{Desirable difficulties}

R. A. Bjork and Bjork (1992) noticed that introducing difficulties during practice often improves memory retention, which they called the desirable difficulties hypothesis. The spacing effect is the most commonly cited desirable difficulty (R. A. Bjork \& Allen, 1970; Cepeda et al., 2009; Cepeda, Pashler, Vul, Wixted, \& Rohrer, 2006): During practice, participants recall fewer items when retrieval practice is spaced apart, rather than massed closely together. However, when tested for long-term recall a few days later, 
this difficulty effect usually reverses-participants recall more spaced items than massed items. Thus, although spaced study increases error rates during training (see, e.g., the rise in Session 2 errors caused by greater Session 2 spacing in Cepeda et al., 2009), it benefits later recall.

Another desirable difficulty is the testing effect, which is that practicing recall, rather than simply restudying, usually improves memory at a delay (Allen, Mahler, \& Estes, 1969; Carpenter, Pashler, Wixted, \& Vul, 2008; Carrier \& Pashler, 1992; Roediger \& Karpicke, 2006). Roediger and Karpicke had participants study prose passages and then either take a practice test (without feedback) or restudy the test material. At a 5-min delay, accuracy was lower in the test condition (75\%) than in the restudy condition $(81 \%)$. However, at greater delays (e.g., 1 week), the effect reversed-participants who had tested (56\%) outperformed those who had restudied (42\%). Again, although testing caused immediate difficulty, it benefited long-term retention.

Other difficulties introduced during practice also benefit memory retention. For instance, randomly mixing conditions (called contextual interference; e.g., ABA-BAB) often impairs immediate memory performance relative to blocking (AAA-BBB), whereas it typically enhances delayed memory performance (Battig, 1966; Shea \& Morgan, 1979). For example, Shea and Morgan had participants learn to knock down freely moveable barriers in three prescribed orders. The blocked group performed one prescribed order, before moving onto the next (e.g., AAA-BBB-CCC). However, the random group performed the prescribed orders randomly by trials (e.g., ABC-CBA-BCA). The random group performed more poorly than the blocked group during acquisition. However, when tested for later retention (e.g., 10 days later), the random group performed the sequences much more quickly $(1.31 \mathrm{~s})$ than did the blocked group (1.73 s).

Desirable difficulties can largely be explained by R. A. Bjork and Bjork's (1992) theory of long-term memory encoding, known as the new theory of disuse (see also E. L. Bjork \& Bjork, 2011; R. A. Bjork, 1994). According to this theory, any given item in long-term memory has both a storage strength and a retrieval strength. Storage strength refers to how well-learned a memory item is, and is assumed to never decline. Retrieval strength refers to how accessible a memory item is, and is assumed to decline over time if the item is not in use. For example, a previous e-mail password or a previous home address might be high in storage strength but low in retrieval strength. In contrast, the order number at a restaurant where you ate lunch would have high retrieval strength but low storage strength.

The new theory of disuse can easily explain why introducing difficulty during practice aids in memory retention: A successful retrieval is assumed to positively increment both storage and retrieval strength. Critically, the permanent increment in storage strength is assumed to be greater if the current retrieval strength is low (i.e., if retrieval is difficult), rather than high. Thus, although retrieval difficulty typically impairs immediate memory performance, the theory makes the (perhaps counterintuitive) prediction that it will benefit future memory performance. Interestingly, most learners appear to be quite unaware of this relationship, readily mistaking temporarily high retrieval strength for high storage strength (Kornell \& Bjork, 2009). Such a metacognitive mistake causes learners to perform poorly on a later test, because they underestimate the need for further study.

To summarize, the desirable-difficulties hypothesis is an attractive empirical generalization that explains a wide variety of effects found in long-term memory research. For practical purposes, however, many of the desirable difficulties identified thus far are inconvenient to implement in the real world. For example, spacing study sessions or varying the conditions of practice complicates the task of instructor and learner alike (cf. Dempster, 1988). Is there an easier way to implement desirable difficulties? For example, could a student obtain a benefit simply by turning on the television or radio while practicing retrieval of an already-established memory trace?

\section{Divided attention and long-term memory}

Previous research has investigated the effects of dividing attention on long-term memory encoding and retention. First, it is well-established that dividing attention during initial memory encoding impairs long-term retention (Anderson \& Craik, 1974; Baddeley, Lewis, Eldridge, \& Thomson, 1984; Craik, Govoni, Naveh-Benjamin, \& Anderson, 1996; Fernandes \& Moscovitch, 2000; Murdock, 1965; NavehBenjamin \& Guez, 2000; for an interesting exception, see Spataro, Mulligan, \& Rossi-Arnaud, 2013). For example, Craik et al. (1996, Exp. 1) had participants encode 15-item word lists and then later perform a free-recall task. During either encoding or recall, participants were asked to perform a secondary continuous reaction time task. In this secondary task, an asterisk could appear in one of four locations and participants pressed a corresponding key, depending on where the asterisk was located. At a later test of long-term retention, fewer words were freely recalled following divided-attention encoding (5.09 words recalled) than following full-attention encoding ( 9.44 words recalled). This finding suggests that dividing attention impairs initial long-term memory encoding.

Many researchers have provided evidence that memory retrieval, like encoding, requires attentional resources, and that dividing attention at retrieval harms performance (Carrier \& Pashler, 1995; Fernandes \& Moscovitch, 2000; Jacoby, 1991; Moscovitch, 1994; Pashler \& Carrier, 1996; Rohrer \& Pashler, 2003; for possible exceptions that occur with certain highly practiced memory retrievals, see Green, 
Johnston, \& Ruthruff, 2011; Hommel, 1998; Logan \& Schulkind, 2000). Carrier and Pashler used a psychological refractory period (PRP) paradigm to assess whether memory retrieval requires central attention. In the PRP paradigm, two speeded tasks are performed, with a variable stimulus-onset asynchrony (SOA) between the two tasks. The classic finding is substantial dual-task slowing on the second task, often attributed to a bottleneck in central processing. Given the existence of a central bottleneck, it is then possible to determine whether any particular processing stage on the secondary task requires the limited-capacity processing mechanism responsible for that bottleneck. Specifically, manipulating the duration of the target process should produce smaller difficulty effects at short than at long SOAs (for a comprehensive discussion of this "locus-of-slack" experimental logic, see Pashler \& Johnston, 1998). Employing this experimental logic, Carrier and Pashler had participants first memorize word pairs either one time (the difficult condition) or five times (the easy condition). Later, participants performed a cued-recall task (Task 2) with a concurrent tone discrimination task (Task 1; high- or low-pitched tone?). The critical finding was additive effects of recall difficulty (word pairs studied one time or five times) and SOA. According to locus-of-slack logic, this indicates that memory retrieval was subject to the central processing bottleneck. To put the point differently, dividing attention caused the memory retrieval on a recognition task to be postponed.

In sharp contrast to research on recognition tasks, some research has suggested (counterintuitively) that free recall is only moderately impaired by dividing attention (e.g., Baddeley et al., 1984; Craik et al., 1996). However, it is possible that the distracting tasks did not place sufficient demands on the central "thought-like" processes required in memory retrieval. For example, Craik et al. used highly compatible stimulus-response mappings in their distracting tasks (e.g., pushing the leftmost button for the leftmost asterisk), which is known to decrease the demands on central attention. Consistent with this account, Rohrer and Pashler (2003), using a similar distracting task with arbitrary (rather than highly compatible) response mappings, demonstrated substantial divided-attention costs on free recall.

Taken together, the evidence suggests that dividing attention does generally impair memory retrieval, especially with only modest levels of retrieval practice. In the experiments reported below, we use divided-attention manipulations known to produce substantial interference during memory recall.

\section{The present study}

As we summarized above, previous research has shown that many types of difficulty instantiated during practice benefit later memory retention. Unlike many previously studied types of desirable difficulty, however, dividing attention has the potential to create desirable difficulty without constraining the scheduling of study. To our knowledge, no previous study has investigated this possibility of a divided-attention "benefit," which is a seemingly logical extension of the desirable difficulties hypothesis.

Given that most dual-task studies have shown negative effects on long-term memory, one might argue that dividing attention is obviously not a desirable difficulty. However, many desirable difficulties (e.g., spacing and testing) produce an immediate performance cost, hence the name desirable "difficulty." It is typically only after delay (e.g., 1-2 days or more) that the pattern reverses and the initial difficulty produces a subsequent benefit. Previous studies of divided attention (e.g., Craik et al., 1996) have almost exclusively tested memory immediately after practice. Thus, it is quite unclear whether a longer retention interval would reveal a dual-task benefit.

On the basis of previous studies, dividing attention at initial encoding would likely not facilitate memory retention; rather, dividing attention should strongly impair encoding. However, dividing attention later in practice, when a memory trace has already been established and needs to be strengthened, might impede memory retrieval while still allowing participants to eventually complete the retrieval; that is, it might create a desirable difficulty that enhances learning. In terms of R. A. Bjork and Bjork's (1992) new theory of disuse, dividing attention may occupy cognitive resources that normally assist in memory retrieval, temporarily lowering memory retrieval strength. Assuming that dividing attention usually does not prevent successful retrieval altogether (i.e., it primarily affects latency), this reduced retrieval strength would lead to a largerthan-usual boost in storage strength.

One study has come close to testing the hypothesis that dividing attention during retrieval practice might benefit long-term retention (although they did not explicitly claim to be testing for a desirable difficulty). Dudukovic, DuBrow, and Wagner (2009) had participants study pictures (for $3 \mathrm{~s}$ each) for later recognition. Next, during retrieval practice, their participants performed a recognition task, either alone or with a concurrent tone discrimination task. When assessed two days later, participants recognized significantly fewer words practiced with divided attention (80\%) than with full attention (89\%) - an effect opposite to a dividedattention benefit. However, we question whether this study provided a fair opportunity for a divided-attention benefit. Pictures are rich in information, and it would be very difficult to encode every detail during a single view of $3 \mathrm{~s}$. Accordingly, the "retrieval practice" sessions provide opportunities for new encoding, which is already known to be harmed by divided attention. As a fair test of the dividedattention benefit, it seems important to use less-detailed study items (e.g., word pairs or succinctly stated facts), for 
which retrieval practice is a relatively pure opportunity for retrieval, not new encoding.

In the present study, participants learned Swahili-English word pairs and were later tested for recall. To maximize the potential benefits of dividing attention, we allowed participants first to establish a memory trace with full attention, before dividing attention. Specifically, after studying each word pair twice and practicing retrieving each English word once with full attention (see Fig. 1), participants again practiced retrieval with full or divided attention. In the full-attention conditions, participants performed a practice test (cued recall) with no additional task. In the dividedattention conditions, they performed a practice test with a concurrent tone-counting task. We anticipated, on the basis of previous research, that divided attention would slow retrieval, and perhaps even result in some retrieval failures, in immediate recall, similar to established desirable difficulties. The critical question, however, was how this retrieval difficulty would affect performance two days later, when participants were tested for memory retention with a cuedrecall task.

\section{Experiment 1}

The goal of this experiment was to determine whether dividing attention while practicing retrieval benefits later memory recall. In Session 1, participants learned 72 Swahili-English word pairs over 12 blocks, with six words per block. Each block consisted of three consecutive phases, shown in Fig. 1: (1) a passive study session, consisting of two presentations of each word pair; (2) a full-attention test (typing the English word corresponding to the presented Swahili word); and then (3) a divided- or full-attention test. The test type in Phase 3 (dual vs. single) alternated between blocks. On divided-attention tests, participants counted the number of high-pitched tones within a sequence of eight tones while a Swahili word appeared on the screen. After the tones ceased, participants responded first to the Swahili word, then to the tone-counting task. Two days later (Session 2), participants received a final cued-recall test for all 72 word pairs under full-attention conditions.

Across participants, the exact same word pairs were used in the divided-attention and full-attention blocks. The critical question was whether dividing attention during memory retrieval creates desirable difficulty. If so, then participants should remember more words from the divided-attention than from the full-attention condition in Session 2.

Method

Participants A group of 46 University of New Mexico students participated for class credit. Five participants were excluded from final analysis because they failed to return for the second session. Another three participants were excluded because of low accuracy on the tone task (less than $70 \%$ ). The resulting 38 participants averaged 19.2 years of age; 18 of them were female.

Apparatus A Dell personal computer presented all visual stimuli on 19-in. CRT monitors and presented all audio stimuli via stereo headphones.

Materials and procedure In Session 1, participants studied the word pairs and practiced recalling them. The word pairs were 72 Swahili-English words (see the Appendix). This word-pair list contained no cognates and no words longer than six letters. Word order was randomized for each participant.

After reading written instructions, participants performed a practice block with three word pairs, familiarizing them with the procedure. Afterward, participants performed the actual experiment of 12 blocks containing six word pairs each. Each block was divided into three phases: (1) a study phase, (2) a full-attention test phase, and (3) a full- or divided-attention test phase (see Fig. 1).

In Phase 1, participants passively studied the word pairs. Each word pair appeared for $5 \mathrm{~s}$, with the Swahili word in white above the English word in red. All word pairs were presented once in a random order, and then presented a second time in a new random order.

In Phase 2, participants performed a full-attention test, intended to establish a strong memory trace before introducing the divided-attention manipulation in Phase 3. Participants had $15 \mathrm{~s}$ to name the English counterpart of the presented Swahili word (which was presented in white). Below the Swahili word was the prompt "English?:" and a gray box in which the participants typed their answer. The response appeared in red as it was typed, and backspaces were allowed. To submit an answer, participants pressed the enter key. If the submitted response was incorrect, an error beep sounded and the correct answer was presented in red below the Swahili word for $4 \mathrm{~s}$. If participants responded correctly, they simply continued to the next trial. During this phase, participants practiced each of the six word pairs once, in a randomized order.

In Phase 3, participants performed another test on each word pair, with either divided or full attention. This was the critical manipulation of the experiment. Full- and dividedattention conditions alternated by blocks, with the block order counterbalanced across participants. In divided-attention blocks, participants performed a concurrent tone task while performing a retention test (see Fig. 1). Participants counted the number of high-pitched tones $(800 \mathrm{~Hz})$ amongst lowpitched tones $(225 \mathrm{~Hz})$. Of the eight total tones, three to six were high tones. The tones were presented for $160 \mathrm{~ms}$, and the 
Fig. 1 Sequences of events on Session 1 of Experiments 1 and 2. In Experiment 1, participants attempted to recall a word while a series of tones played. In Experiment 2, participants were actually forced to respond to the word prompt before the tones ceased
Events within a Block on Day 1 (12 blocks total)

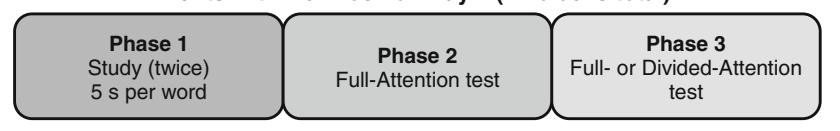

(A)

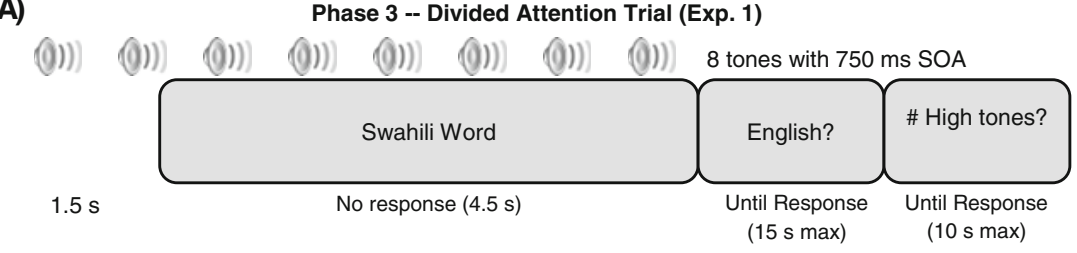

(B)

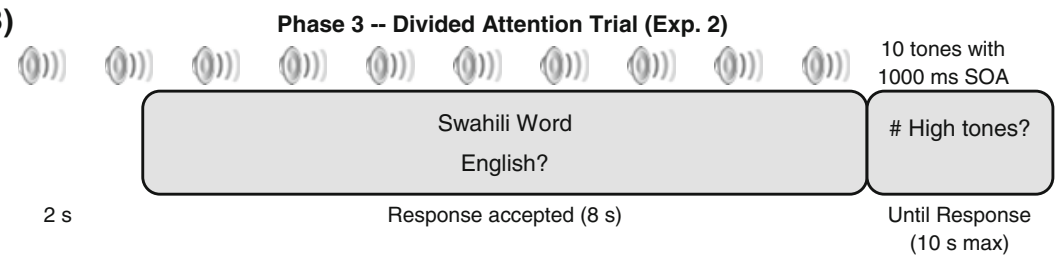

onsets of the tones were spaced $750 \mathrm{~ms}$ apart. The Swahili word cue appeared simultaneously with the onset of the third tone in the sequence. After the tones had ceased, participants were prompted to respond to the presented Swahili word $(15 \mathrm{~s}$ maximum). If this word response was incorrect, they received feedback displaying the correct answer (4 s). Then, they were prompted to respond to the tone task ( $10 \mathrm{~s}$ maximum) with the message "Number of high tones?" Participants typed their answer into a gray box. If the response was incorrect, the participant received immediate feedback displaying the correct number of tones. At the end of the block, participants received feedback about their accuracy in that block. If participants' tone task accuracy was too low in a block (below $80 \%$ ), they received a warning advising them to focus more on tone task accuracy.

The timing within the full-attention condition in Phase 3 was yoked to the timing within the divided-attention condition. Each trial began with a blank screen $(1.5 \mathrm{~s})$. Then, participants viewed the Swahili word for $4.5 \mathrm{~s}$, mimicking the time spent during tone presentation in the dividedattention condition. Finally, participants were allowed to respond to the word task ( $15 \mathrm{~s}$ maximum). If they responded incorrectly, they received feedback. To mimic the time it took to type the response to the tone task in the dividedattention condition (estimated from pilot data), participants viewed a blank screen for $1.5 \mathrm{~s}$ before beginning the next full-attention trial. This yoking ensured that the spacing of retrieval (known to be an important factor) was nearly identical in the full- and divided-attention conditions.

Session 2 occurred roughly $48 \mathrm{~h}$ after Session 1 . In this session, participants completed a cued-recall task followed by a recognition task. The cued-recall task was similar to that in Phase 2 of Session 1. Participants were presented with a Swahili word and asked to type its English counterpart. Participants had $45 \mathrm{~s}$ to respond and were encouraged to continue trying to retrieve the English word until time ran out, rather than simply pressing the Enter key and skipping to the next trial. After participants had responded, they were told whether that response was incorrect, but were not told the correct answer. Participants completed 12 blocks of six word pairs each, in a random order. No performance feedback was presented at the end of each block, though participants were allowed to take a break. The recognition task in Session 2 was similar to a multiple-choice test. Participants were presented with a Swahili word in white along with four English words in red, labeled 1 through 4. The three foil words were other English words that had been studied in Session 1. Participants responded by pressing keys labeled "1," "2," "3," and "4" (the actual keys were 1, 2, 8, and 9, respectively). The participants were told whether each response was correct or incorrect, but were not told the correct answer.

\section{Results and discussion}

Mean accuracy is shown in Table 1. First, preliminary $t$ tests were conducted on the Session 1 data. Recall improved from $76.6 \%$ in Phase 2 (always full attention) to $86.8 \%$ in Phase 3 (full or divided attention), $t(37)=8.34, p<.001$. In Phase 3 , recall was not significantly worse in the divided-attention condition $(85.7 \%)$ than in the full-attention condition $(88.0 \%), t(37)=1.61, p>.10$. However, participants typed in correct answers more slowly under divided attention (3.7 s) than under full attention (2.1 s), $t(37)=14.37, p<$ .001 . This latency effect (a slowing of $80 \%$ ) indicates that our manipulation of retrieval difficulty was successful.

A two-way analysis of variance (ANOVA) with the factors Session (Phase 3 of Session 1 vs. Session 2) and Attention Condition (divided vs. full) was conducted on cued-recall accuracy. Recall accuracy was much higher in Session 1 
Table 1 Percentages correct (PC) and standard errors of the means $(S E)$ for by session and phase for Experiment 1

\begin{tabular}{|c|c|c|c|c|c|c|c|}
\hline & \multicolumn{3}{|l|}{ Session 1} & \multicolumn{4}{|c|}{ Session 2} \\
\hline & \multirow[b]{2}{*}{ Phase 2} & \multicolumn{2}{|l|}{ Phase 3} & \multicolumn{2}{|c|}{ Cued Recall } & \multicolumn{2}{|c|}{ Recognition } \\
\hline & & Divided & Full & Divided & Full & Divided & Full \\
\hline $\mathrm{PC}$ & $76.6 \%$ & $85.7 \%$ & $88.0 \%$ & $15.0 \%$ & $16.1 \%$ & $61.3 \%$ & $61.5 \%$ \\
\hline$S E$ & $2.1 \%$ & $1.7 \%$ & $1.5 \%$ & $1.4 \%$ & $1.8 \%$ & $3.0 \%$ & $2.4 \%$ \\
\hline
\end{tabular}

In Phase 3, participants performed a practice test under full or divided attention. In Session 2, we assessed recall for word pairs previously practiced - in Session 1-under full or divided attention

$(86.8 \%)$ than in Session $2(15.5 \%)$, indicating dramatic memory loss, $F(1,37)=1,196.298, M S E=.010, p<.001$, $\eta_{\mathrm{p}}{ }^{2}=.982$. We observed no main effect of attention, $F(1,37)=$ $2.404, M S E=.005, p>.10, \eta_{\mathrm{p}}{ }^{2}=.061$; the memory loss across sessions did not significantly differ between the divided-attention (70.7\%) and full-attention (71.9\%) conditions, $F(1,37)<1, M S E=.004, p>.10, \eta_{\mathrm{p}}{ }^{2}=.010$.

The critical question was whether practicing retrieval while dividing attention would improve memory retention. If so, in Session 2, participants should have recalled more words practiced with divided than with full attention. Preplanned $t$ tests revealed no such divided-attention benefit in Session 2, in either the cued-recall task (15.0\% divided vs. $16.1 \%$ full), $t(37)<1$, $p>.10$, or the recognition task (61.3\% divided vs. $61.5 \%$ full), $t(37)<1, p>.10$. Although we found no absolute benefit of prior practice with divided attention (suggesting no practical benefit), for theoretical reasons it was worth asking whether successful retrievals with divided attention in Session 1 are especially resistant to forgetting. Analyzing only words that were successfully retrieved in Session 1, we still found no benefit in Session 2 of divided attention (16.4\%) relative to full attention $(16.9 \%), t(37)<1, p>.10$. Similarly, on the cuedrecall task, participants did not correctly answer words practiced under divided attention (5.4 $\mathrm{s}$ ) faster than words practiced under full attention (5.4 s), $t(34)<1, p>.10$ (note: three participants could not be included in this analysis due to an absence of correct responses in one condition or the other in Session 2).

To summarize, we tested whether dividing attention during retrieval practice represents a desirable difficulty that improves memory retention after a delay. Participants responded much more slowly in the divided-attention condition than in the fullattention condition of Session 1 (by about $80 \%$ ), indicating that retrieval was difficult. However, dividing attention was not "desirable," in that it did not produce any benefit in Session 2 on either cued recall or a recognition task.

\section{Experiment 2}

In Experiment 1, we observed no long-term benefit of dividing attention during retrieval practice for memory retention.
However, it is logically possible that some participants tried to avoid actually dividing attention in Session 1. That is, participants could have waited for the entire tone sequence to finish before beginning memory retrieval with full attention. This seems unlikely to have occurred frequently, given that recall latencies were delayed by only a few seconds; however, we cannot rule out the possibility that it occurred some of the time. In Experiment 2, therefore, we implemented a "forced" dual-tasking, in which participants had to respond to the word task during the sequence of tones.

\section{Method}

Participants A new set of 49 University of New Mexico students volunteered for class credit. Four participants were excluded from final analysis because they failed to return for the second session. One participant was excluded because of low accuracy on the tone task in Session 1 (less than $70 \%$ of trials with a reported tone count within one of the actual count). Of the remaining 44 participants, the average age was 20.9 years, and 32 were female.

Apparatus, materials, and procedure The methods were identical to those of Experiment 1, except that the participants now had to respond to the word before the tones ceased (see Fig. 1B). To allow adequate time to respond to the word, we used ten tones (instead of eight, as in Exp. 1). Of the ten total tones, three to seven were high tones. Because pilot participants had low tone-counting accuracy, we increased the SOA between the tones from 750 to 1,000 ms. Again, the timing of the full-attention condition was yoked to the timing of the divided-attention condition. A blank screen appeared for $2 \mathrm{~s}$ followed by the Swahili-English word pair for $8 \mathrm{~s}$. Then an additional blank screen appeared for $1.5 \mathrm{~s}$, to compensate for the average time taken to respond to the tone task. Again, this full-attention yoking assured that any observed benefit in the divided-attention condition would not merely reflect greater spacing between items. A final change is that the recognition task was dropped from Session 2, as it did not seem to provide results different from those in the (more traditional) cuedrecall task. 


\section{Results and discussion}

Mean accuracy rates are displayed in Table 2. Preliminary $t$ tests were conducted on the Session 1 data. Cued-recall accuracy increased from $71.9 \%$ in Phase 2 (full attention only) to $80.0 \%$ in Phase 3 (full and divided attention), $t(43)=6.66, p$ $<.001$. In Phase 3, recall accuracy was slightly lower in the divided-attention condition $(78.0 \%)$ than in the full-attention condition $(82.0 \%), t(43)=2.56, p<.05$. On trials in which participants correctly answered the cued-recall task, they did so more slowly in the divided-attention $(3.6 \mathrm{~s})$ than in the fullattention $(3.0 \mathrm{~s})$ condition, $t(43)=8.07, p<.001$.

Accuracy was lower on the tone-counting task in this experiment $(77.8 \%)$ than in Experiment $1(88.2 \%), t(80)=3.92$, $p<.001$. Presumably, this decrement resulted from the "forced" dual-tasking in this experiment: Participants had to retrieve and type the English word during the tone sequence. However, the mean lenient tone accuracy (responses within \pm 1 of the actual tone count were considered "correct") was $96.8 \%$, suggesting that participants were genuinely attempting to count the tones.

The same two-way ANOVA from Experiment 1 was conducted on recall accuracy, with the variables Session (Phase 3 of Session 1 vs. Session 2) and Attention Condition (divided vs. full). Recall accuracy was higher in Session 1 $(80.0 \%)$ than in Session $2(17.0 \%)$, indicating memory loss, $F(1,43)=685.381, M S E=.026, p<.001, \eta_{\mathrm{p}}{ }^{2}=.941$. Recall accuracy was not significantly different in the dividedattention condition $(47.6 \%)$ and the full-attention condition $(49.4 \%), F(1,43)=2.471, M S E=.006, p>.10, \eta_{\mathrm{p}}{ }^{2}=.054$. Memory loss was significantly less for the divided-attention words $(60.8 \%)$ than for the full-attention words $(65.3 \%), F(1$, $43)=5.045, M S E=.004, p<.05, \eta_{\mathrm{p}}{ }^{2}=.105$; note that this significant effect stemmed from the fact that the dividedattention condition started at a lower accuracy in Session 1, and thus had "less to lose."

The critical question was whether dividing attention during retrieval practice improved later memory retention. If so, we would expect participants to recall more divided-attention

Table 2 Percentages correct (PC) and standard errors (SE) by session and phase for Experiment 2

\begin{tabular}{|c|c|c|c|c|c|}
\hline & \multicolumn{3}{|l|}{ Session 1} & \multirow{2}{*}{\multicolumn{2}{|c|}{ Session 2}} \\
\hline & \multirow[b]{2}{*}{ Phase 2} & \multicolumn{2}{|l|}{ Phase 3} & & \\
\hline & & Divided & Full & Divided & Full \\
\hline PC & $71.9 \%$ & $78.0 \%$ & $82.0 \%$ & $17.2 \%$ & $16.7 \%$ \\
\hline$S E$ & $2.7 \%$ & $2.8 \%$ & $2.6 \%$ & $1.9 \%$ & $1.8 \%$ \\
\hline
\end{tabular}

In Phase 3, participants performed a practice test under full or divided attention. In Session 2, we assessed recall for word pairs previously practiced - in Session 1-under full or divided attention. words than full-attention words in Session 2. Preplanned $t$ tests revealed no such divided-attention benefit on Session 2 (17.2\% divided vs. $16.7 \%$ full), $t(43)<1, p>.10$. Again, a more sensitive test for a divided-attention benefit would be to only look at retention of words successfully recalled on Phase 3 of Session 1. Even in this analysis, we found no significant benefit for divided-attention words $(20.1 \%)$ relative to full-attention words $(18.6 \%), t(43)<1, p>.10$. Similarly, participants did not recall words practiced under divided attention $(4.9 \mathrm{~s})$ faster than words practiced under full attention $(4.9 \mathrm{~s}), t(41)<1, p\rangle$ .10 (note: two participants were excluded, due to $0 \%$ accuracy in the full- or the divided-attention word condition).

In summary, we used a modified procedure designed to force participants to retrieve the English word from the Swahili cue while counting tones. Nevertheless, participants still showed no divided-attention benefit on Session 2 recall.

\section{Experiment 3}

In Experiments 1 and 2, we found no evidence that dividing attention during retrieval practice created a desirable difficulty that improved memory retention. In both experiments, overall recall accuracy was relatively low in Session $2(16.3 \%)$. This poor performance likely reflected presenting only six word pairs per block, creating relatively massed practice (rather than spaced). We reasoned that such conditions were ideal for observing a benefit of dividing attention, since it is wellestablished that additional massed full-attention practice would produce little benefit. We attempted to take an otherwise easy memory retrieval (due to massed practice) and create the necessary retrieval difficulty by dividing attention. Clearly, this did not happen. Nevertheless, the memory retrievals in Phase 3 might have been so easy that even the divided-attention manipulation could not raise difficulty sufficiently, precluding any divided-attention benefit.

In Experiment 3, we addressed this issue by tripling the number of words per block (from six to 18 words). The extra spacing between the presentations of each word pair should make Phase 3 retrieval more difficult, which might give dividing attention a better opportunity to produce a benefit. It should also place overall Session 2 memory performance in a much higher range, reducing any concerns about floor effects.

Method

Participants A new set of 54 University of New Mexico students participated for class credit. Eight participants were excluded from final analysis because they failed to return for the second session. Three participants were excluded because of low accuracy on the tone task in Session 1 (less than $70 \%$ with lenient accuracy scoring in which a count 
within \pm 1 was considered correct). Three other participants were excluded because of abnormally low accuracy on Phase 2 of Session 1 (less than $10 \%$ ), suggesting that they were not following instructions. Of the remaining 40 participants, the average age was 20.1 years old, and 28 were female.

Apparatus, materials, and procedure All of the stimuli and procedures were identical to those of Experiment 2, except that participants now studied 18 word pairs per block instead of six. This meant that there were only four blocks total per session, instead of 12 .

\section{Results and discussion}

Mean accuracy is shown in Table 3. Recall accuracy improved from $49.9 \%$ in Phase 2 (full attention only) to $56.1 \%$ in Phase 3 (full and divided attention), $t(39)=4.49, p<.001$. In Phase 3 , recall accuracy was lower in the divided-attention condition $(53.4 \%)$ than in the full-attention condition $(58.9 \%), t(39)=$ $2.63, p<.05$. When they were accurate on the cued-recall task, participants responded more slowly in the divided-attention condition (4.2 s) than in the full-attention condition (3.6 s), $t(39)=4.62, p<.001$. As in Experiment 2, the forced dualtasking in the divided-attention condition caused low tonecounting accuracy (68.1\%). However, the mean lenient tone accuracy ( \pm 1 tone) was $93.3 \%$, suggesting that participants were actually dual-tasking but often missed a tone.

As in the previous experiments, a two-way ANOVA with the factors Session (Phase 3 of Session 1 vs. Session 2) and Attention Condition (full vs. divided) was conducted on the mean recall accuracy. Recall accuracy was higher in Session 1 $(56.1 \%)$ than in Session $2(31.0 \%)$, indicating some forgetting, $F(1,39)=181.066, M S E=.014, p<.001, \eta_{\mathrm{p}}{ }^{2}=.823$. Recall accuracy was marginally lower in the divided-attention $(42.0 \%)$ than in the full-attention $(45.1 \%)$ condition, $F(1,39)=3.856, M S E=.009, p=.057, \eta_{\mathrm{p}}{ }^{2}=.090$. The amount of memory loss was significantly less in the dividedattention condition $(22.7 \%)$ than in the full-attention condition $(27.6 \%), F(1,39)=4.547, M S E=.005, p<.05, \eta_{\mathrm{p}}{ }^{2}=.104$.

Table 3 Percentages correct (PC) and standard errors (SE) for the cued-recall task by session and phase for Experiment 3

\begin{tabular}{|c|c|c|c|c|c|}
\hline & \multicolumn{3}{|c|}{ Session 1} & & \\
\hline & \multirow[b]{2}{*}{ Phase 2} & \multicolumn{2}{|l|}{ Phase 3} & \multicolumn{2}{|l|}{ Session 2} \\
\hline & & Divided & Full & Divided & Full \\
\hline PC & $49.8 \%$ & $53.4 \%$ & $58.9 \%$ & $30.7 \%$ & $31.3 \%$ \\
\hline$S E$ & $3.0 \%$ & $3.0 \%$ & $2.7 \%$ & $2.4 \%$ & $2.6 \%$ \\
\hline
\end{tabular}

In Phase 3, participants performed a practice test under full or divided attention. In Session 2, we assessed recall for word pairs previously practiced - in Session 1 - under full or divided attention.
However, this result largely derived from the lower baseline in the divided-attention condition in Session 1.

The key question in this experiment was whether practicing retrieval with divided-attention would benefit later recall. Overall, Session 2 accuracy was much higher in this experiment $(31.0 \%)$ than in Experiment $2(17.0 \%)$, replicating the classic spacing effect, $t(82)=4.82, p<.001$. Despite this boost in overall Session 2 performance, participants still did not remember divided-attention words $(30.7 \%)$ better than fullattention words $(31.2 \%), t(39)<1, p>.10$. As a more sensitive test for a divided-attention benefit, we analyzed only words that had been successfully retrieved in Phase 3 of Session 1. Still, participants did not remember a greater percentage of the divided-attention words $(42.4 \%)$ than full-attention words $(44.0 \%), t(39)<1, p>.10$. Participants were also not significantly slower to correctly answer divided-attention words $(5.0 \mathrm{~s})$ than full-attention words $(4.8 \mathrm{~s}), t(39)<1, p>.10$.

In summary, participants practiced more words per block in this experiment, to provide more opportunity for participants to benefit from retrieval during Phase 3 of Session 1 and to boost overall accuracy on Session 2 (taking performance further from the floor). The extra spacing of items on Session 1 in this experiment, relative to Experiment 2, did in fact nearly double recall in Session 2. This finding argues against the claim that a floor effect had occurred in the previous experiments, since the extra spacing was still able to produce a very large benefit. However, dividing attention again did not improve memory retention.

\section{General discussion}

The desirable difficulties hypothesis characterizes an important trade-off that is widespread in human memory (Allen et al., 1969; R. A. Bjork \& Allen, 1970; Carrier \& Pashler, 1992; Cepeda et al., 2009, 2006; Craik, 1970; Gardiner, Craik, \& Bleasdale, 1973; Roediger \& Karpicke, 2006). In the present study, we asked whether dividing attention can also represent a desirable difficulty, offering a useful tool in real-world contexts. Dividing attention often impairs performance on a secondary task, and there is evidence that memory retrievals are no exception (especially when they are not highly practiced; Carrier \& Pashler, 1995; Fernandes \& Moscovitch, 2000; Jacoby, 1991; Moscovitch, 1994; Pashler \& Carrier, 1996; Rohrer \& Pashler, 2003). So, it seemed reasonable to expect that dividing attention might also create a desirable difficulty during retrieval practice, and thereby benefit memory retention. In terms of the new theory of disuse, dividing attention might temporarily decrease retrieval strength, generating an especially large boost in storage strength (R. A. Bjork \& Bjork, 1992).

In our experiments, participants learned 72 SwahiliEnglish word pairs. Half of the word pairs were practiced 
while performing an irrelevant tone task (divided attention), whereas the other half were practiced with no additional task (full attention). Two days later, participants performed a final memory recall test. In Experiment 1, participants studied six words per block and, in the divided-attention condition, recalled words after the tones had ceased. Although dividing attention prolonged recall latencies in Session 1, it did not improve retention in Session 2 on recall or recognition performance (see Fig. 2). In Experiment 2, we employed "forced" dual-tasking in the divided-attention condition, in which participants had to respond to the word prompt before the tones ceased. Again, dividing attention did not lead to a memory benefit in Session 2. Finally, in Experiment 3, we sampled a much higher performance range in Session 2 by increasing the number of words presented per block from six to 18 in Session 1. The resulting increase in Session 1 spacing nearly doubled the overall retention in Session 2, replicating many previous studies of the spacing effect (R. A. Bjork \& Allen, 1970; Cepeda et al., 2009; Cepeda et al., 2006). However, participants still showed no divided-attention benefit in Session 2.

Thus, although we sampled a range of different conditions across the three experiments (recall vs. recognition, spaced vs. massed practice, different dual-task manipulations), all three suggested that dividing attention during retrieval practice does not improve memory retention. Pooling the data from all three experiments, the $95 \%$ confidence interval about the dividedattention "benefit" on percentages of recall in Session 2 was $0.4 \pm 1.8$; this means that we can rule out even a very small benefit.

The present experiments failed to produce a dividedattention benefit in learning, despite our deliberately attempting to establish the most favorable conditions for eliciting such a benefit. In Session 1, we divided attention only after participants developed a strong memory trace; at this point, a successful full-attention memory retrieval should have been relatively easy (due to high retrieval strength), and therefore have produced little strengthening of memory. In addition, the divided-attention condition was devised to provide

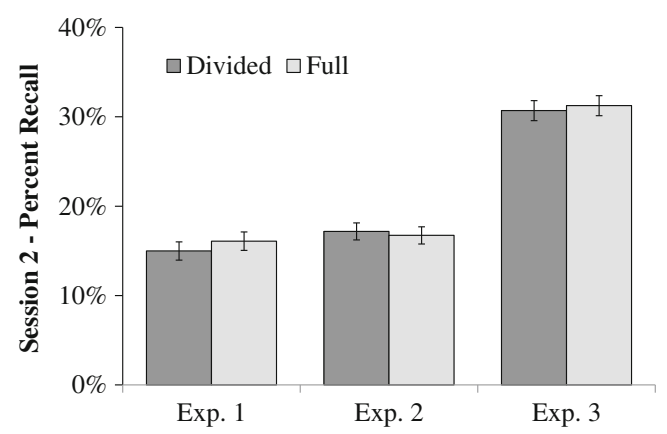

Fig. 2 Mean percentages correct in the cued-recall task of Session 2, by experiments, for full-attention and divided-attention words. Error bars represent the standard errors of the means, based upon the mean squared error of the interaction between attention condition and session sufficient time for memory retrieval, so that participants might struggle but would typically succeed at recall. Nonetheless, no benefit of divided attention was observed in Session 2. We speculate that dividing attention without our "optimal" design features would likely have resulted in greatly reduced memory retention. Thus, divided attention is generally an undesirable difficulty from the standpoint of memory retention; it causes extra effort and time on the part of the learner during retrieval practice, but fails to provide a compensatory benefit.

Although the present study found that dividing attention during memory retrieval failed to increase subsequent memory retention (in Session 2), that does not mean that dividing attention had no effect on performance at all. In Session 1, we found clear signs that retrievals were impaired under divided attention (i.e., it did represent a genuine "difficulty"). Pooling the data from Phase 3 of Session 1 of all three experiments, recall was worse in the divided-attention conditions $(72.3 \%)$ than in the full-attention conditions $(76.3 \%), t(121)=$ $4.00, p<.001,95 \%$ confidence interval (CI) $[-3.9 \pm 1.9]$. In addition, when the response was correct, participants responded more slowly in the divided-attention condition ( $3.8 \mathrm{~s})$ than in the full-attention condition $(2.9 \mathrm{~s})$, a $31 \%$ increase in latencies that was statistically significant, $t(121)=$ $12.29, p<.001,95 \% \mathrm{CI}[0.9 \pm 0.14]$. Hence, our data support the conclusion of previous research that memory retrieval requires central attentional resources, and is thus subject to divided-attention costs (Carrier \& Pashler, 1995; Fernandes \& Moscovitch, 2000; Jacoby, 1991; Moscovitch, 1994; Pashler \& Carrier, 1996; Rohrer \& Pashler, 2003). These experiments were not designed to test claims that divided attention harms encoding more than retrieval, so the present data are silent on that issue (Baddeley et al., 1984; Craik et al., 1996).

\section{What makes a difficulty desirable?}

Some types of difficulties during training - such as spacing, testing, and contextual interference - reliably improve later memory retention. However, other difficulties - such as dividing attention - do not seem to benefit memory retention. What is the key difference between difficulties that improve and those that harm memory retention?

The desirable difficulties hypothesis is primarily an empirical generalization that need not, by itself, necessarily suggest any particular mechanism. R. A. Bjork and Bjork's (1992) new theory of disuse, however, asserts that a desirable difficulty is one that temporarily decreases retrieval strength. In turn, this decrement in retrieval strength allows for a larger boost in permanent storage strength. Consider, for example, spacing. If a learner masses study, he or she can rely on a temporarily high retrieval strength (R. A. Bjork \& Allen, 1970). Spacing study, on the other hand, allows retrieval strength to decay. This low retrieval strength allows for an especially large increase in storage strength upon 
successful retrieval (note: there are other accounts of spacing effects than the desirable difficulties account, including contextual variability or differential rehearsal).

Undesirable difficulties, such as dividing attention, may be those manipulations that do not reduce retrieval strength. This account is consistent with other accounts proposing that difficulties that are irrelevant to memory consolidation - such as visually obscuring to-be-remembered words (Yue, Castel, \& Bjork, 2013; but see Mulligan, 1996), withholding feedback during retrieval practice (Pashler, Cepeda, Wixted, \& Rohrer, Pashler et al. 2005), or a drastic discrepancy between visual onscreen text and auditory narration (Yue, Bjork, \& Bjork, 2013) - do not benefit later retention. In the present study, dividing attention may have merely delayed retrieval during practice, rather than making it more difficult per se. This claim is consistent with studies contending that memory retrieval cannot occur concurrently with other cognitive processes (Carrier \& Pashler, 1995). In the present study, the primary tone task may have used up all available processing resources, effectively blocking memory retrieval. Participants may have rapidly switched between the memory recall and the tone tasks (increasing mean reaction times on the word task). This strategy would result in full retrieval strength during recall, offering no subsequent benefit on memory retention. Our use of two different types of concurrent tasks (across experiments) was designed to give us extra chances to find a difficulty level well-suited to producing a memory benefit; however, if parallel processing is generally impossible, then perhaps no divided-attention condition will actually yield an intermediate retrieval strength.

\section{Limitations and directions for future research}

Naturally, it cannot be ruled out that some type of dividedattention manipulation besides the ones investigated here would constitute a desirable difficulty. Although we deliberately set out to create favorable conditions for finding a benefit, we obviously did not sample all possible dividedattention manipulations. Future research could explore other types of divided-attention demands, such as ones that produce greater task confusion or tasks that incorporate stimuli designed to produce associative interference (harkening back to the early experiments of Battig, 1966).

\section{Concluding remarks}

Many studies have demonstrated that memory retrieval difficulty introduced during learning leads to memory benefits during later retrieval (R. A. Bjork \& Bjork, 1992). On the basis of this empirical generalization from a diverse set of manipulations and contexts, it seemed plausible that divided attention might also provide such a benefit. However, all three of our experiments produced no divided-attention benefit, despite our deliberate attempts to create conditions promoting such a benefit. We did, however, replicate the usual finding of a large benefit of spacing rather than massing practice. The present findings raise the question of why many types of difficulties at practice (e.g., spacing and testing) do improve long-term retention, but dividing attention does not. One speculation is that difficulty benefits performance only when it reduces retrieval strength to an intermediate level. Instead, dividing attention may merely delay the onset of the retrieval, without actually reducing retrieval strength.

Author note Nicholas Gaspelin and Eric Ruthruff, Department of Psychology, University of New Mexico; Harold Pashler, Department of Psychology, University of California - San Diego.

This work was supported by a collaborative activity award to H. Pashler from the J.S. McDonnell Foundation, a MURI award from the Office of Naval Research (25684A), and by grants from the Institute of Education Sciences (Grant R305B070537 to H. Pashler) and NSF (Grant SBE0542013, G.W. Cottrell, PI). We thank Henry L. Roediger, III, Fergus Craik, Neil Mulligan and James S. Nairne for their valuable comments.

Correspondence concerning this article should be addressed to Nicholas Gaspelin, Department of Psychology, 1 University of New Mexico, MSC03 2220, Albuquerque, NM 87131-1161.E-mail: gaspelin@unm.edu.

\section{Appendix}

Table 4 Our 72 Swahili-English words pairs (alphabetized by Swahili word)

\begin{tabular}{|c|c|c|c|c|c|}
\hline Swahili & English & Swahili & English & Swahili & English \\
\hline alama & sign & gongo & top & mapwa & beach \\
\hline andiko & book & guba & bay & masia & walk \\
\hline asali & honey & hakimu & judge & mimba & fruit \\
\hline bahari & sea & hamali & load & mofa & bread \\
\hline bakora & stick & hasho & plug & moyo & heart \\
\hline banda & barn & hasidi & enemy & munda & board \\
\hline bati & metal & hatua & step & nafasi & room \\
\hline chama & group & hela & money & nakawa & sound \\
\hline chapeo & hat & hindi & corn & pevu & adult \\
\hline chengo & camp & karamu & party & ramba & cloth \\
\hline cheti & note & kasha & box & rubani & guide \\
\hline chondo & bag & kidoto & cup & safu & line \\
\hline dahabu & gold & kigoli & girl & shamba & farm \\
\hline dawati & desk & kinoo & soap & shimo & well \\
\hline dindi & hole & kipua & nose & siku & day \\
\hline dunia & world & kitalu & fence & tama & cheek \\
\hline duwara & wheel & kiti & chair & tariki & road \\
\hline farasi & horse & kodi & $\operatorname{tax}$ & tuza & prize \\
\hline fora & goal & kondoo & sheep & umbo & shape \\
\hline fumo & chief & kota & curve & utando & film \\
\hline futari & meal & liwado & trail & vuvi & snake \\
\hline galili & shell & lukuma & food & wardi & rose \\
\hline gebali & rock & malalo & bed & watani & home \\
\hline gereza & jail & maliki & king & zomeo & cry \\
\hline
\end{tabular}




\section{References}

Allen, G. A., Mahler, W. A., \& Estes, W. K. (1969). Effects of recall tests on long-term retention of paired associates. Journal of Verbal Learning and Verbal Behavior, 8, 463-470.

Anderson, C. M., \& Craik, F. I. M. (1974). The effect of a concurrent task on recall from primary memory. Journal of Verbal Learning and Verbal Behavior, 13, 107-113.

Baddeley, A., Lewis, V., Eldridge, M., \& Thomson, N. (1984). Attention and retrieval from long-term memory. Journal of Experimental Psychology. General, 113, 518-540. doi:10.1037/0096-3445.113.4.518

Battig, W. F. (1966). Facilitation and interference. In E. A. Bilodeau (Ed.), Acquisition of skill (pp. 215-244). New York, NY: Academic Press.

Bjork, E. L., \& Bjork, R. A. (2011). Making things hard on yourself, but in a good way: Creating desirable difficulties to enhance learning. In M. A. Gernsbacher, R. W. Pew, L. M. Hough, \& J. R. Pomerantz (Eds.), Psychology and the real world: Essays illustrating fundamental contributions to society (pp. 56-64). New York, NY: Worth.

Bjork, R. A. (1994). Memory and metamemory considerations in the training of human beings. In J. Metcalfe \& A. Shimamura (Eds.), Metacognition: Knowing about knowing (pp. 185-205). Cambridge, MA: MIT Press.

Bjork, R. A., \& Allen, T. W. (1970). The spacing effect: Consolidation or differential encoding. Journal of Verbal Learning and Verbal Behavior, 9, 567-572.

Bjork, R. A., \& Bjork, E. L. (1992). A new theory of disuse and an old theory of stimulus disfluctuation. In A. Healy, S. Kosslyn, \& R. M. Shiffrin (Eds.), From learning processes to cognitive processes: Essays in honor of William K. Estes (Vol. 2, pp. 35-67). Hillsdale, NJ: Erlbaum.

Carpenter, S. K., Pashler, H., Wixted, J. T., \& Vul, E. (2008). The effects of tests on learning and forgetting. Memory \& Cognition, 36, 438-448. doi:10.3758/MC.36.2.438

Carrier, M., \& Pashler, H. (1992). The influence of retrieval on retention. Memory \& Cognition, 20, 633-642.

Carrier, M., \& Pashler, H. (1995). Attentional limits in memory retrieval. Journal of Experimental Psychology: Learning, Memory, and Cognition, 21, 1339-1348.

Cepeda, N. J., Coburn, N., Rohrer, D., Wixted, J. T., Mozer, M. C., \& Pashler, H. (2009). Optimizing distributed practice: Theoretical analysis and practical implications. Experimental Psychology, 56, 236-246.

Cepeda, N. J., Pashler, H., Vul, E., Wixted, J. T., \& Rohrer, D. (2006). Distributed practice in verbal recall tasks: A review and quantitative synthesis. Psychological Bulletin, 132, 354-380. doi:10.1037/00332909.132.3.354

Craik, F. I. M. (1970). The fate of primary memory items in free recall. Journal of Verbal Learning and Verbal Behavior, 9, 143-148.

Craik, F. I. M., Govoni, R., Naveh-Benjamin, M., \& Anderson, N. D. (1996). The effects of divided attention on encoding and retrieval processes in human memory. Journal of Experimental Psychology. General, 125, 159-80.

Dempster, F. N. (1988). The spacing effect: A case study in the failure to apply the results of psychological research. American Psychologist, 43, 627-634. doi:10.1037/0003-066X.43.8.627

Dudukovic, N. M., DuBrow, S., \& Wagner, A. D. (2009). Attention during memory retrieval enhances future remembering. Memory \& Cognition, 37, 953-961. doi:10.3758/MC.37.7.953

Fernandes, M. A., \& Moscovitch, M. (2000). Divided attention and memory: Evidence of substantial interference effects at retrieval and encoding. Journal of Experimental Psychology. General, 129, $155-176$.

Gardiner, J. M., Craik, F. I. M., \& Bleasdale, F. A. (1973). Retrieval difficulty and subsequent recall. Memory \& Cognition, 1, 213-216.
Green, C., Johnston, J. C., \& Ruthruff, E. (2011). Attentional limits in memory retrieval-Revisited. Journal of Experimental Psychology. Human Perception and Performance, 37, 1083-1098.

Hommel, B. (1998). Automatic stimulus-response translation in dualtask performance. Journal of Experimental Psychology. Human Perception and Performance, 24, 1368-1384. doi:10.1037/00961523.24.5.1368

Jacoby, L. L. (1991). A process dissociation framework: Separating automatic from intentional uses of memory. Journal of Memory and Language, 30, 513-541. doi:10.1016/0749-596X(91)90025-F

Kornell, N., \& Bjork, R. A. (2009). A stability bias in human memory: Overestimating remembering and underestimating learning. Journal of Experimental Psychology. General, 138, 449-468. doi:10. 1037/a0017350

Logan, G. D., \& Schulkind, M. D. (2000). Parallel memory retrieval in dual-task situations: I. Semantic memory. Journal of Experimental Psychology: Human Perception and Performance, 26, 10721090. doi:10.1037/0096-1523.26.3.1072

Moscovitch, M. (1994). Cognitive resources and divided attention interference effects at retrieval in normal people: The role of the frontal lobes and medial temporal cortex. Neuropsychology, 8, 524-534.

Mulligan, N. W. (1996). The effects of perceptual interference at encoding on implicit memory, explicit memory, and memory for source. Journal of Experimental Psychology: Learning, Memory, and Cognition, 22, 1067-1087. doi:10.1037/0278-7393.22.5.1067

Murdock, B. B., Jr. (1965). Effects of a subsidiary task on short-term memory. British Journal of Psychology, 56, 413-419.

Naveh-Benjamin, M., \& Guez, J. (2000). Effects of divided attention on encoding and retrieval processes: Assessment of attentional costs and a componential analysis. Journal of Experimental Psychology: Learning, Memory, and Cognition, 26, 1461-1482.

Pashler, H., Cepeda, N. J., Wixted, J. T., \& Rohrer, D. (2005). When does feedback facilitate learning of words? Journal of Experimental Psychology: Learning, Memory, and Cognition, 31, 3-8.

Pashler, H., \& Carrier, M. (1996). Structures, processes, and the flow of information. In E. L. Bjork \& R. A. Bjork (Eds.), Memory (Handbook of Perception and Cognition (2nd ed., pp. 3-29). San Diego, CA: Academic Press.

Pashler, H., \& Johnston, J. C. (1998). Attentional limitations in dualtask performance. In H. Pashler (Ed.), Attention (pp. 155-189). Hove, UK: Psychology Press.

Roediger, H. L., III, \& Karpicke, J. D. (2006). Test-enhanced learning: Taking memory tests improves long-term retention. Psychological Science, 17, 249-255. doi:10.1111/j.1467-9280.2006.01693.x

Rohrer, D., \& Pashler, H. E. (2003). Concurrent task effects on memory retrieval. Psychonomic Bulletin \& Review, 10, 96-103.

Schmidmaier, R., Ebersbach, R., Schiller, M., Hege, I., Holzer, M., \& Fischer, M. R. (2011). Using electronic flashcards to promote learning in medical students: Retesting versus restudying. Medical Education, 45, 1101-1110.

Shea, J. B., \& Morgan, R. L. (1979). Contextual interference effects on the acquisition, retention, and transfer of a motor skill. Journal of Experimental Psychology: Human Learning and Memory, 5, 179-187.

Spataro, P., Mulligan, N. W., \& Rossi-Arnaud, C. (2013). Divided attention can enhance memory encoding: The attentional boost effect in implicit memory. Journal of Experimental Psychology: Learning, Memory, and Cognition. Advance online publication. doi:10.1037/a0030907

Yue, C. L., Bjork, E. L., \& Bjork, R. A. (2013). Reducing verbal redundancy in multimedia learning: An undesired desirable difficulty? Journal of Educational Psychology. Advance online publication. doi:10.1037/a0031971

Yue, C. L., Castel, A. D., \& Bjork, R. A. (2013b). When disfluency is - and is not - a desirable difficulty: The influence of typeface clarity on metacognitive judgments and memory. Memory \& Cognition, 41, 229-241. doi:10.3758/s13421-012-0255-8 